\title{
Combination of Gabor and Curvelet Texture Features for Face Recognition Using Principal Component Analysis
}

\author{
Shafin Rahman, Sheikh Motahar Naim, Abdullah Al Farooq, and Md. Monirul Islam
}

\begin{abstract}
Biometric face Recognition is a new generation technology for identification and verification. Many techniques have been developed for this purpose in past two decades. A good face recognition technique must require unique, effective and efficient features from face images. Content based image retrieval (CBIR) can extract features that can be useful for face recognition. Recently, Gabor and curvelet texture features have been successfully used in image retrieval research. In this paper, we propose a novel face recognition method that uses texture features obtained by calculating mean and standard deviation of Gabor and curvelet transformed face images. PCA is then applied to the feature vectors instead of entire transformed images which traditional methods do. Using this process, we build four classifiers using mean and standard deviation calculated from Gabor and curvelet transformed face images. For identification purpose, a new matching strategy is proposed that checks goodness of four matching results of the classifiers. As we consider only mean or standard deviation features, the image representation has comparatively lower dimensions. Furthermore, our proposed method does not necessarily require all the input images to be of same resolution. We evaluate the proposed method using ORL and Yale face databases. The recognition results of the experiments show that our approach is significantly better than the conventional methods.
\end{abstract}

Index Terms - Content based image retrieval (CBIR); gabor and curvelet transform; principal component analysis (PCA).

\section{INTRODUCTION}

We can recognize faces within a second that is a great demonstration of incredible human intelligence but this must be a very difficult problem for a computer [1]. Over the last two decades, researchers are making their best effort for achieving high performance rate in automated face recognition system. It is an interesting and challenging research field in biometrics and computer vision and is extensively used in security system, human-computer interface, surveillance and so on.

To-date, researchers have proposed a number of face recognition approaches [1]. Most of them use Principal Component Analysis (PCA). PCA is a powerful tool for extracting some characteristic information of the dataset to identify pattern. It performs dimensionality reduction to input dataset and represents the whole face-space in lower dimension that contains most variance to highlight similarities and differences within the face-classes. PCA

Manuscript received May 17, 2012; revised June 26, 2012.

The authors are with the Department of Computer Science and Engineering Bangladesh University of Engineering and Technology(BUET) Dhaka- 1000, Bangladesh based approaches [3], [4] consider only raw pixel intensity of the face images. Furthermore, these approaches require that the input face images must be ideally aligned and under well-controlled illumination. To overcome these shortcomings, a number of approaches transform images by different filters like Gabor [5], [6] and curvelet [8], [9] and then apply PCA. However, they still use the raw pixel intensity of transformed images for face recognition. Such process inevitably implies high processing time and memory requirement for large dimensional feature vector.

Due to face image is an unstructured array of pixels, we believe that the first step in understanding the face is to extract efficient and effective visual features from these pixels. There are thousands of publications in the literature describing color, texture, shape and spatial feature extraction for image retrieval [2]. But most of the approaches of face recognition consider only pixel intensity values of different transformation of images like Gabor [5], [6] or curvelet [8], [9] transformation. Raw pixel intensity values cannot be a good feature for face representation due to the curse of high dimensionality and semantic gap which is the difference between the high level interpretation of a face image and its low level pixel representation. The use of content based image retrieval (CBIR) could solve this problem [10], [11]. Content features like Gabor and curvelet texture can capture appropriate features having strong discriminative capability in image retrieval and semantic learning techniques. Being inspired from the success of image retrieval, we firstly use a new face image representation method that obtains texture features found by calculating the mean and standard deviation of the Gabor filtered or curvelet transformed images for face recognition. Although both Gabor and curvelet can capture face information efficiently, those features do not find same information because of having different spectrum in frequency domain. Therefore, it may happen that Gabor misses to capture some significant discriminative information where curvelet succeeds or vice versa. For this reason, we propose a new technique to combine those features efficiently that enable to function features independently. Final decision is generated by checking the goodness of performance of individual features. Moreover, our proposed method solves high dimensionality problem of image representation efficiently. Furthermore, a new flexibility is inserted in our approach that it can work properly although all input images have different dimensionality. In our proposed method, we first divide the face image into sub-images. After that, Gabor and curvelet texture features are extracted from the sub-images to feed into PCA and create four different classifiers. Each classifier can find an intermediate match using nearest neighbor 
method. For similarity measure to identify test faces with the gallery face images, we propose a match based decision making system named final decision machine (FDM) that calculates the final match from the outputs of individual classifiers.

The remaining parts of this paper are organized as follows. Section II describes Gabor and curvelet feature extraction. The combination process of Gabor and curvelet feature is described in Section III. In Section IV, the proposed face recognition system is explained. Section $\mathrm{V}$ describes image databases and presents experimental results, followed by conclusion and discussion in Section VI.

\section{II.FEATURE EXTRACTION PROCESS}

\section{A. Extracting Gabor Features}

For a given image $I(x, y)$ with size $\Psi_{a, b, \theta}(x, y)$, its discrete Gabor transform is given by a convolution:

$$
G_{m n}(x, y)=\sum_{s} \sum_{t} I(x-s, y-t) \Psi_{m n}^{*}(s, t)
$$

where, $s$ and $t$ are the filter mask size variable and $\Psi_{m n}$ is the complex conjugate of $\Psi_{m n}$ which is a class of self-similar functions generated from dilation and rotation of the following mother wavelet:

$$
\Psi(x, y)=\frac{1}{2 \pi \sigma_{x} \sigma_{y}} \exp \left[-\frac{1}{2}\left(\frac{x^{2}}{\sigma_{x}}+\frac{y^{2}}{\sigma_{y}}\right)\right] \cdot \exp (j 2 \pi W x)
$$

where, $W$ is called the modulation frequency. The self-similar Gabor wavelets are obtained through the generating function:

$$
\Psi_{m n}(x, y)=a^{-m} \Psi(\tilde{x}, \tilde{y})
$$

where, $m$ and $n$ specify the scale and orientation of the wavelet respectively with

$$
\begin{aligned}
m=0,1, . ., M-1, h=, 1, \ldots, N-1 \text { and } \\
\tilde{x}=a^{-m}(x \cos \theta+y \sin \theta) \\
\tilde{y}=a^{-m}(-x \sin \theta+y \cos \theta)
\end{aligned}
$$

where, $a>1$ and $\theta=h \pi N$ and $M$ and $N$ are the total number of scale and orientation used. We use 5 scales and 8 orientations and get 40 Gabor filters. For each convoluted image, we calculate a mean and a standard deviation. Thus we obtain two 40 dimensional feature vectors of mean and standard deviation for a face image representation [10].

\section{B. Extracting Curvelet Features}

The concept of curvelet transform has been extended from the two-dimensional ridgelet transform. Given an image $I(x, y)$ of dimension $P \times Q$, its digital curvelet transform at scale $a$, translation $b$ and orientation $\theta$ is defined as,

$$
C T^{D}(a, b, \theta)=\sum_{x=0}^{P-1} \sum_{y=0}^{Q-1} I\left(\begin{array}{ll}
x & y
\end{array}\right) \stackrel{D}{D} \underset{a, b, \theta}{ } \Psi x \quad y(
$$

where, the superscript $D$ stands for digital implementation of the curvelet transform. The 2D ridgelet function $\Psi_{a, b, \theta}(x, y)$ is generated from a univariate function, $\Psi(x, y)$ which has vanishing mean and sufficient decay. The ridgelet $\Psi_{a, b, \theta}(x, y)$ is given as,

$$
\Psi_{a, b, \theta}(x, y)=a^{1 / 2} \Psi((x \cos \theta+y \sin \theta-b) / a)
$$

A ridgelet is a wavelet type function and is constant along the lines: $x \cos \theta+y \sin \theta=$ const .

After obtaining the coefficients in $C T^{D}(a, b, \theta)$, the mean and standard deviation are calculated from each set of curvelet coefficients. Each image is decomposed into 4 scales that creates $26(=1+8+16+1)$ sub-bands. Thus we generate two 26 dimensional feature vectors of mean and standard deviation, calculated from those scales and orientations [11].

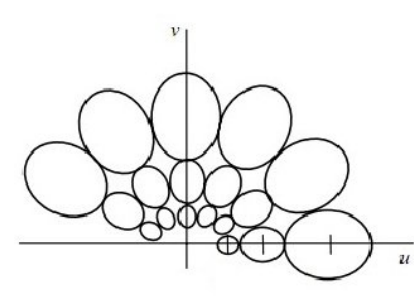

(a)

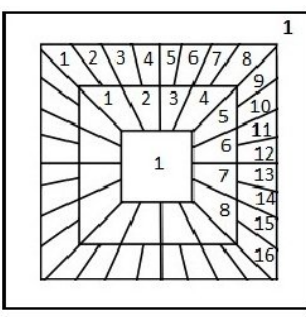

(b)
Fig.1. Frequency spectrum coverage by (a) Gabor and (b) curvelet.

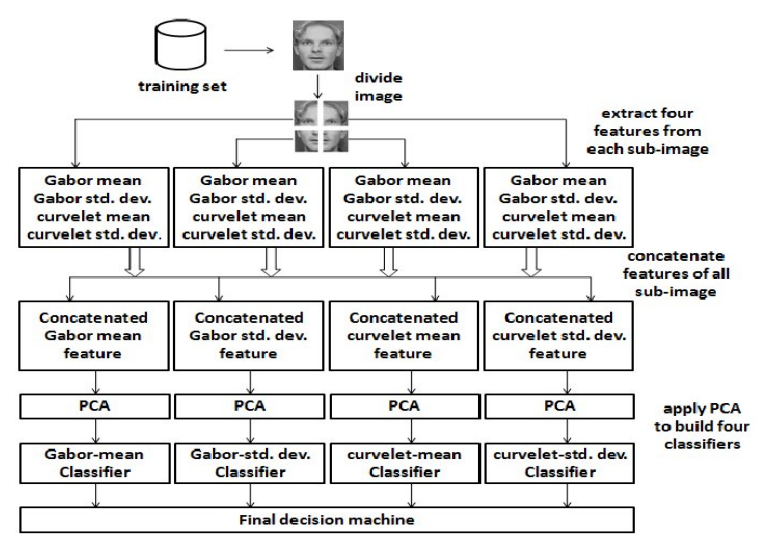

Fig. 2. Training flow of proposed approach

\section{COMBINING GABOR AND CURVELET FEATURES}

As face is a non-rigid object, it deforms its shape due to facial expression, body movement and changes its appearance in different illumination. A good face recognition technique should work robustly being independent of varying expression and illumination. To adapt the system with such problems, we need to consider local information of faces. Content feature like Gabor and curvelet can extract such information in different scales and orientations. But, here, one significant consideration is that high degree 
abstraction of pixel intensity values of face images to low level Gabor or curvelet features must lose some characteristic information which we call semantic gap. Moreover, as Gabor and curvelet do not covers exactly same spectrum in frequency domain (see Fig. 1), there must be some information that remains in curvelet but not in Gabor features and vice versa. Although it is proved that curvelet features contain more accurate information than Gabor [11], these two methods treat a face image differently. In Fig. 1, we see that as there are some holes in frequency spectrum of Gabor, it covers only a part of the spectrum. In contrary, curvelet covers the spectrum completely. So, curvelet covers more accurate edge or line information than Gabor. On the other hand, Gabor features are useful if face image has mostly uniform texture [10]. Thus, it may happen, for a given face, curvelet feature can be able to identify the face but not Gabor feature or vice versa.

For this reason, our motivation is that combination of Gabor and curvelet should work more efficiently than considering those individually. Such idea is used in [7] by combining modular PCA and wavelet PCA. In our case, we can combine features by concatenation. But there are some problems of such approach. Firstly, some part of frequency spectrum will overlap with one another i.e. some information will be considered multiple times that certainly we do not desire. Secondly, it will increase the dimensionality of feature vector. To solve problems of combining different features working together, we propose to let different features work independently and then check the goodness of performance of individual features to decide the final match. Moreover, we have divided the faces in different grids or sub-images so that it improves the performance of feature by capturing more local information [4], [6], [7].

\section{Proposed Algorithm}

\section{A. Training}

We assume that the input face is a 2D array of gray scale pixel intensity values. So, for colored face, we need to convert it from RGB to gray scale. We further assume that the input image must contain only the face region. So, in the preprocessing step, we need to crop the face region from images. Fig. 2 shows the training flow of our system. The training system includes three stages. At first, we extract features from face to compute four representations. After that, four different classifiers are built using those four representations. Finally, testing the system with the training faces to fix some selections such as: best classifier, threshold values and required number of sub-images. All this parameters will be used in testing phase. Threshold values are necessary to measure of the goodness of classifiers performance.

Feature representation: At first, we divide the face into grid wise sub-images. The number of sub-images depends on the feature and the database being used.

We do experiment for four different number of sub-images: $1(=1 \times 1), ?(=2 \times 2), ?(=3 \times 3)$ and $?(=4 \times 4)$.For each sub-image, we apply Gabor transform and extract mean feature vectors as described in Section II(A). Then, concatenating feature vectors of all sub-images, we make feature representation of the entire face. If there is $p$ number of sub-images, the feature vector of entire face will have $p \times 40$ dimension. We call it Gabor mean feature vector. Similarly, we calculate Gabor standard deviation feature vector. Like Gabor features, we calculate curvelet mean feature vector and curvelet standard deviation feature vector of the entire face using the process described in Section II(B). Each of these four feature vectors can represent the face individually:

$$
\begin{gathered}
f_{\text {mean }}^{z}=\left[\mu_{11} \mu_{12}, \ldots, \mu_{1 q}, \mu_{21}, \mu_{22}, \ldots, \mu_{2 q}, \ldots, \mu_{p q}\right] \\
f_{\text {std }}^{z}=\left[\gamma_{11} \sigma_{12}, \ldots, \sigma_{1 q}, \sigma_{21}, \sigma_{22}, \ldots, \sigma_{2 q}, \ldots, \sigma_{p q}\right]
\end{gathered}
$$

where, $z$ represents Gabor or curvelet features, $p=$ no. of sub-images, $q$ = total no. of features extracted from a sub-image; $\mu_{i j}$ and $\sigma_{i j}$ denote $j$-th mean and standard deviation feature of the $i$-th sub-image respectively and $i=1$, $2, \ldots p$ and $j=1,2, \ldots q$. One important benefit of such feature vectors is that the number of features extracted from face does not depend on image resolution. So, in our approach all the face images do not need to be of same resolution.

Building classifier with PCA: We obtain four feature vectors for a single face image. Using these feature vectors, we build four different classifiers by PCA [3]: Gabor mean classifier, Gabor standard deviation classifier, curvelet mean classifier and curvelet standard deviation classifier. The procedure of building a classifier is discussed below.

Suppose, $\Gamma_{1}, \Gamma_{2}, \Gamma_{3}, \ldots, \Gamma_{L}$ are the column vectors representing any of the four features of $L$ training face images. The average of those feature vectors is $\Psi=(1 / L) \sum_{n=1}^{L} \Gamma_{n}$. So, the difference from original feature vector to average feature vector is defined by the vector, $\Phi_{n}=\Gamma_{n}-\Psi$. Let, $\Phi=\left[\Phi_{1} \Phi_{2} \ldots \Phi_{\mathrm{L}}\right]$ is subject to PCA that finds certain orthogonal eigenvectors that best describe the distribution. Suppose, $u_{n}$ and $\lambda_{n}$ are the eigenvector and eigenvalue of the covariance matrix,

$$
C=\frac{1}{L} \sum_{n=1}^{L} \Phi_{n} \Phi_{n}^{T}
$$

The eigenvectors and eigenvalues of the covariance matrix are calculated. Eigenvectors of highest eigenvalues encode larger variation in the training feature vector set. Next, $\Phi_{n}$ is projected on the face space by the following operation:

$$
\gamma_{n}=u_{n}^{T} \Phi_{\mathrm{n}}
$$

where, $n=1,2, \ldots . L$ The representative feature vector for $i$-th face class, $\Omega_{i}$ is described by averaging the result of eigenvector representation over face images. Then, the 
feature vector of testing face image $\left(\Gamma_{\text {test }}\right)$ is projected on the face space by the operation: $\left.\omega_{n}=\mathfrak{u}_{n}^{T} \Gamma_{\text {test }}-\Psi\right)$, where $\Omega_{\text {test }}^{T}=\left[\omega_{1} \omega_{2} \ldots \omega_{L}\right]$ is the contribution of each eigenvector to the feature vector of a input image. $\Omega^{\text {test }}$ is used for the pattern recognition task to find which face class is the best described for an input image. For similarity measure, nearest neighbor calculated by Euclidean distance is used to find the best match class.

$$
\varepsilon_{i}=\left\|\Omega_{\text {test }}-\Omega_{i}\right\|
$$

where, $\Omega_{i}$ is the vector describing the $i$-th face class. A face is classified to a face class $c$ where $\epsilon_{i}$ is minimum.

$$
c=i_{\text {min }}=\arg \min _{\mathrm{i}}\left(\varepsilon_{i}\right)
$$

The outputs of classifying a face are: the best intermediate match and minimum Euclidean distance associated with that match.

Selection of best classifier, threshold and number of sub-images required: All selections will be done while testing the system with the training faces. Among the four classifiers, the best one is that which has maximum recognition rate in training example. Next, we need to find threshold values. These values are used to check goodness of the matches of classifiers. The procedure of the threshold selection is as follows:

From the description of the previous subsection, we see that when a face is classified by a classifier, it gets an intermediate match and a Euclidean distance. Suppose, among $L$ faces in the training database, $L_{r}$ faces are rightly classified by a particular classifier. Let, $\varepsilon_{1}, \varepsilon_{2}, \varepsilon_{3} \ldots \varepsilon_{L r}$ the distances measured for $L_{r}$ faces. The threshold $(T)$ for this classifier is the average of those Euclidean distances.

$$
T=\frac{1}{L_{r}} \sum_{k=1}^{L_{r}} \varepsilon_{k}
$$

Thus, we get different threshold values for four classifiers. Each threshold value denotes the predicted measurement of distance to classify a face for a specific classifier. Then, experimenting this process with different number of sub-images of training faces, we find the required number of sub-image for which a particular classifier works most efficiently. All of these selection parameters will be used by FDM.

\section{B. Testing}

As described in previous section, each of our individual classifiers is able to classify a new face instance. To classify a new face image, we send it to each of the four classifiers separately. Before that, we need to extract features from the face by exactly same way as used in building individual

\begin{tabular}{|c|c|c|c|c|c|c|c|c|c|c|c|c|}
\hline \multirow{3}{*}{ Sub-image } & \multicolumn{6}{|c|}{ ORL database } & \multicolumn{6}{|c|}{ Yale database } \\
\hline & \multicolumn{3}{|c|}{ Gabor } & \multicolumn{3}{|c|}{ curvelet } & \multicolumn{3}{|c|}{ Gabor } & \multicolumn{3}{|c|}{ curvelet } \\
\hline & $\operatorname{Dim}^{n}$ & $\begin{array}{c}\text { Mean } \\
(\%)\end{array}$ & $\begin{array}{l}\text { StD. } \\
(\%)\end{array}$ & $\operatorname{Dim}^{n}$ & $\begin{array}{c}\text { Mean } \\
(\%)\end{array}$ & $\begin{array}{l}\text { StD. } \\
(\%)\end{array}$ & $\operatorname{Dim}^{n}$ & $\begin{array}{c}\text { Mean } \\
(\%)\end{array}$ & $\begin{array}{l}\text { StD. } \\
(\%)\end{array}$ & $\operatorname{Dim}^{n}$ & $\begin{array}{c}\text { Mean } \\
(\%)\end{array}$ & $\begin{array}{l}\text { StD. } \\
(\%)\end{array}$ \\
\hline 1 & 40 & 80 & $\begin{array}{c}80.8 \\
3 \\
\end{array}$ & 26 & 57.50 & $\begin{array}{c}60.8 \\
3 \\
\end{array}$ & 40 & 68.89 & $\begin{array}{c}73.3 \\
3 \\
\end{array}$ & 26 & 62.22 & $\begin{array}{c}75.5 \\
6 \\
\end{array}$ \\
\hline 4 & 160 & 86.67 & 85 & 104 & 81.67 & $\begin{array}{c}83.3 \\
3\end{array}$ & 160 & 86.67 & $\begin{array}{c}77.7 \\
8\end{array}$ & 104 & 75.56 & $\begin{array}{c}82.2 \\
2\end{array}$ \\
\hline 9 & 360 & 85.83 & $\begin{array}{c}84.1 \\
7 \\
\end{array}$ & 234 & 93.33 & $\begin{array}{c}93.3 \\
3 \\
\end{array}$ & 360 & 66.67 & $\begin{array}{c}77.7 \\
8 \\
\end{array}$ & 234 & 75.56 & $\begin{array}{c}86.6 \\
7 \\
\end{array}$ \\
\hline 16 & 640 & 90.83 & $\begin{array}{c}83.3 \\
3\end{array}$ & 416 & 83.33 & 95 & 640 & 42.22 & $\begin{array}{c}71.1 \\
1\end{array}$ & 416 & 80 & $\begin{array}{c}88.8 \\
9\end{array}$ \\
\hline
\end{tabular}

TABLE I: RECOGNITION RATE OF INDIVIDUAL CLASSIFIER WHILE TRAINING EXAMPLE BASED ON TESTING IN DIFFERENT SUB-IMAGES AND FEATURE DIMENSION

classifier. Each classifier gives an intermediate match and a Euclidean distance. Match results, Euclidean distances are sent to Final Decision Machine (FDM). FDM is a match based decision making system that checks the goodness of the intermediate matches. Traditionally, other face recognition techniques need not to use such decision criteria because there are no intermediate matches associated with those methods. On the other hand, our approach classifies a face by four independent ways so that it is necessary to decide the best final match among the intermediate matches. Suppose, $c_{1}, c_{2}, c_{3}, c_{4}$ are intermediate matches and $\varepsilon_{1}, \varepsilon_{2}, \varepsilon_{3}, \varepsilon_{4}$ are the Euclidean distances of the corresponding match for four classifiers respectively. From the training stage, the best classifier and threshold values of classifiers are also fixed. Let, $T_{1}, T_{2}, T_{3}, T_{4}$ are the four threshold values. The FDM works as follows:

- The match having majority votes of the classifiers among the four intermediate matches will be the final match trivially.

- If any of the two classifiers give one match and other two classifiers give another match i.e. $\left(c_{1}=c_{2}\right) \cap\left(c_{3}=c_{4}\right)$ or $\left(c_{1}=c_{3}\right) \cap\left(c_{2}=c_{4}\right)$ or $\left(c_{1}=c_{4}\right) \cap\left(c_{2}=c_{3}\right)$ then the match given by the best classifier will be the final match. 
- If four different classifiers give four different match i.e. $\left(c_{1} \neq c_{2} \neq c_{3} \neq c_{4}\right)$ then we need to compare the associated Euclidean distances of the matches with the threshold values. For this, we calculate the ratio $\left(R_{h}\right)$ of Euclidean distances $\varepsilon_{h}^{\text {test }}$ to threshold values $T_{h}$ of corresponding classifiers by this operation.

$$
R_{h}=\frac{\varepsilon_{h}^{t e s t}}{T_{h}}
$$

Here, $h=1,2,3,4$. As threshold values are the predicted distances of the classifiers so ideally we expect the ratio should be 1 . So, the match given by the classifier for which this ratio gets close to 1 is the final match.

\section{IMAGE DATABASE AND EXPERIMENTAL RESULT}

To evaluate the performance of our algorithm, we choose ORL [13] and Yale [14] face databases. The ORL database consists of 40 individuals and 10 gray-scale images per subject. Yale face database contains 165 gray-scale images of 15 individuals and 11 images per subject. These images are of different facial expression and lighting condition. Fig. 3 shows some sample images of ORL and Yale databases.

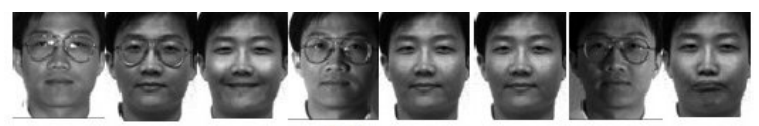

(a)

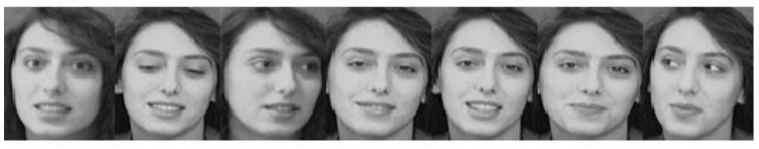

(b)

Fig. 3. Sample faces from (a) Yale (b) ORL database

We choose two third faces from database randomly for training purpose and rest face images are used as testing. At the preprocessing stage, we crop the face region from the image using Viola-Jones Fast Face Detection Mex Implementation [12]. As our system doesn't need all the faces to be of same resolution, we do not need to resize the face images. After extracting four feature vectors from faces, we build four different classifiers to train our system. Then, we perform training example based testing. By this testing, we are able to fix the best classifier and threshold values. Now, experimenting with different number of sub-images, we fix the required number of sub-images that works best for individual classifier. Table I shows the performance of each classifier in different number of sub-images and feature dimension of such training example based testing. Here, we can see that Gabor mean classifier works efficiently at $4 \times 4$ sub-images in ORL database while $2 \times 2$ sub-images is the best for the same classifier in Yale database. It further shows that the dimension of different feature vectors is low. This enables faster computation and low memory requirement.

For testing purpose, we pass the test face to each of the four different classifiers. To classify a face, each classifier outputs an intermediate match and a Euclidian distance associated with that match. Then, FDM decides the final match comparing the confidence of the matches of each classifier. In Table II, we show the performance of our method. Here, we also compare the result with individual classifiers performance. The performance of individual classifiers is lower than the combination of classifiers. This is because; one classifier may fail to recognize a face where other classifier succeeds. Thus, it's useful to work the classifiers jointly to ensure maximum recognition rate. So, the combination of Gabor and curvelet features work more efficiently than considering them individually.

TABLE II: RECOGNITION RATE OF ORL AND YALE DATABASES ON TEST

\begin{tabular}{|c|c|c|c|c|c|}
\hline \multirow[b]{3}{*}{ Database } & \multirow{2}{*}{\multicolumn{2}{|c|}{ Gabor }} & \multirow{2}{*}{\multicolumn{2}{|c|}{ curvelet }} & \multirow{3}{*}{$\begin{array}{r}\text { Our } \\
\text { method } \\
(\%)\end{array}$} \\
\hline & & & & & \\
\hline & $\begin{array}{c}\text { Mean } \\
(\%)\end{array}$ & $\begin{array}{l}\text { StD. } \\
(\%)\end{array}$ & $\begin{array}{c}\text { Mean } \\
(\%)\end{array}$ & $\begin{array}{l}\text { StD. } \\
(\%)\end{array}$ & \\
\hline ORL & 88.57 & 83.33 & 92.86 & 93.33 & 98.33 \\
\hline Yale & 77.78 & 73.33 & 75.56 & 86.67 & 95.56 \\
\hline
\end{tabular}

Our proposed method achieves comparatively better performance than traditional approaches on ORL and Yale databases. Table III shows comparison of some other methods with our approach. Yale database is not used in [4], [6].

TABLE III: COMPARISON WITH OTHER PAPERS EXPERIMENT.

\begin{tabular}{ccccc}
\hline Database & $\begin{array}{c}\text { SH-PCA } \\
{[4](\%)}\end{array}$ & $\begin{array}{c}\text { Modular PCA } \\
{[6](\%)}\end{array}$ & $\begin{array}{c}\text { Improved } \\
\text { MPCA [7] }(\%)\end{array}$ & $\begin{array}{c}\text { Our } \\
\text { method } \\
(\%)\end{array}$ \\
\hline ORL & 90 & 88.9 & 96.22 & 98.33 \\
Yale & - & - & 89.17 & 95.56 \\
\hline
\end{tabular}

For a particular number of sub-images, we need to pass the training faces two times: one for building classifiers and other for number of sub-images, best classifier and threshold selection. Moreover, to identify a face we need to pass it to each of the four classifiers. Thus, one can claim that it will increase the order of computation. But, practically, all of such computation will be done in offline. On the other hand, each of our classifier can work independently in parallel. So, this technique is very useful for face recognition.

\section{CONCLUSION}

In this paper, we combine Gabor and curvelet texture features to work together for face recognition. We find that texture features not only reduce the dimensionality but also improve the performance significantly. The paper further shows that those features capture the face characteristics efficiently better than raw pixel. As Gabor and curvelet features recognize faces at different confidence level, we propose a match based decision making system called FDM that checks the goodness of different features for final match. Unlike traditional approaches, our proposed method does not apply PCA on each sub-image. After finding required number of sub-images, we just apply PCA only once for each classifier. Low dimension texture features extracted from the sub-images represent the image-space that is much smaller than previous PCA based approach. For those reasons, our 
computational complexity and memory requirement is low. We use ORL and Yale face databases for our experiment and get comparatively better result than other PCA based approaches. In future work, rotation and scale invariant Gabor and curvelet feature will be investigated to further improve the performance face recognition.

\section{REFERENCES}

[1] W. Zhao, R. Chellappa, P. J. Phillips, and A. Rosenfeld. Face recognition: A literature survey, ACM Comput. Surv, vol. 35, no. 4, pp. $399-458,2000$

[2] R. Datta, D. Joshi, J. Li, and J. Z. Wang, Image Retrieval: Ideas, Influences, and Trends of the New Age, ACM Computing Surveys, vol. 40, no. 2, pp. 1-60, 2008

[3] M. A. Turk and A. P. Pentland, Eigenfaces for recognition, Journal of Cognitive Neuroscience, vol. 3, no. 1, pp. 71-86, 1991.

[4] M. M. Khan, M. Y. Javed, and M. A. Anjum, Face Recognition Using Sub-Holistic PCA, Information and Communication Technologies, pp. 152-157, 2005.

[5] K. Cung, S. C. Kee, and S. R. Kim, Face Recognition Using Principal Component Analysis of Gabor Filter Responses, In Proc. of the International Workshop on Recognition, Analysis, and Tracking of Faces and Gestures in Real-Time Systems, pp. 53, 1999.

[6] N. Gudur and V. Asari, Gabor Wavelet Based Modular PCA Approach for Expression and Illumination Invariant Face Recognition, Applied Imagery and Pattern Recognition Workshop, 35th IEEE, pp. 13, Oct. 2006.

[7] W. Puyati and A. Walairacht, Efficiency Improvement for Unconstrained Face Recognition by Weightening Probability Values of Modular PCA and Wavelet PCA, Advanced Communication Technology, vol. 2, pp. 1449-1453, 2008.

[8] T. Mandal, Q. M. J. Wu and Y. Yuan, Curvelet based face recognition via dimension reduction, Signal Processing, vol. 89, no. 12, pp. 2345-2353, 2009.

[9] J. Xie, Face Recognition Based on Curvelet Transform and LS-SVM, in Proc. of the 2009 International Symposium on Information Processing, pp. 140-143, 2009.

[10] D. Zhang and A. Wong, M, Indrawan and G. Lu. Content-based Image Retrieval Using Gabor Texture Features, The First IEEE Pacific-Rim Conference on Multimedia, pp. 13-15, 2000.

[11] I. J. Sumana, M. M. Islam, D. Zhang and G. Lu, Content based image retrieval using curvelet transform, In Proc. of 2008 International Workshop on Multimedia Signal Processing, pp. 11-16, 2008.

[12] P. Viola and M. Jones, Rapid Object Detection using a Boosted Cascade of Simple Features, Computer Vision and Pattern Recognition, vol. 1, pp. 511, 2001.
[13] F. Samaria and A. Harter, Parameterisation of a Stochastic Model for Human Face Identification, Proceedings of 2nd IEEE Workshop on Applications of Computer Vision, Sarasota FL; Dec. 1994.

[14] P. N. Bellhumer, J. Hespanha, and D. Kriegman, Eigenfaces vs. fisherfaces: Recognition using class specific linear projection, IEEE Transactions on Pattern Analysis and Machine Intelligence, Special Issue on Face Recognition, vol. 17, no. 7, pp. 711-720; 1997.

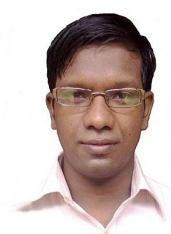

Shafin Rahman was born in Rangpur, Bangladesh in 1988. He is a B.Sc.student of the department of Computer Science and Engineering, Bangladesh University of Engineering and Technology(BUET). In coherence with his being a devoted researcher in the field of computer vision, image processing and pattern recognition, he has a special focus of interest in face recognition technology.

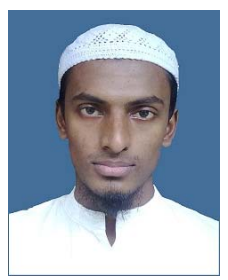

Sheikh Motahar Naim was born in Chittagong, Bangladesh in 1987. He is currently pursuing his B.Sc. degree in the department of Computer Science and Engineering at Bangladesh University of Engineering and Technology(BUET). His research interest includes computer vision, human-computer interaction, image processing with a special focus on face recognition technology.

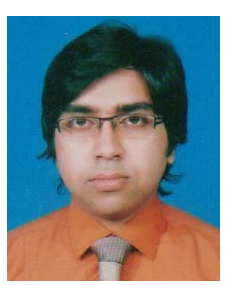

Abdullah Al Farooq was born in Mymensing, Bangladesh in 1987. He is a B.Sc.student of the department of Computer Science and Engineering at Bangladesh University of Engineering and Technology(BUET). His research interest includes computer vision, image processing and specially face recognition technology.

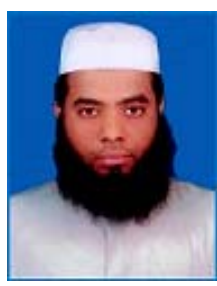

Md. Monirul Islam received the B. Sc Engineering degree and M. Sc. Engineering degree in Computer Science and Engineering (CSE) from Bangladesh University of Engineering and Technology (BUET) in 2001 and 2004, respectively and Ph.D. from Monash University, Australia in 2009. He joined the Department of Computer Science and Engineering, BUET in 2002. His current research interests include image retrieval, computer vision and pattern recognition. He is currently an assistant professor in the Department of CSE, BUET. 